\title{
Existence of Solutions to Fractional Mixed Integrodifferential Equations with Nonlocal Initial Condition
}

\author{
A. Anguraj, ${ }^{1}$ P. Karthikeyan, ${ }^{2}$ and J. J. Trujillo ${ }^{3}$ \\ ${ }^{1}$ Department of Mathematics, PSG College of Arts and Science, Coimbatore 641 014, India \\ ${ }^{2}$ Department of Mathematics, KSR College of Arts and Science, Tiruchengode 637 215, India \\ ${ }^{3}$ Departamento de Análisis Matemático, Universidad de La Laguna, Tenerife 38271 La Laguna, Spain
}

Correspondence should be addressed to J. J. Trujillo, jtrujill@ullmat.es

Received 14 November 2010; Accepted 6 January 2011

Academic Editor: Dumitru Baleanu

Copyright (C) 2011 A. Anguraj et al. This is an open access article distributed under the Creative Commons Attribution License, which permits unrestricted use, distribution, and reproduction in any medium, provided the original work is properly cited.

\begin{abstract}
We study the existence and uniqueness theorem for the nonlinear fractional mixed Volterra-Fredholm integrodifferential equation with nonlocal initial condition $d^{\alpha} x(t) / d t^{\alpha}=f(t, x(t)$, $\left.\int_{0}^{t} k(t, s, x(s)) d s, \int_{0}^{1} h(t, s, x(s)) d s\right), x(0)=\int_{0}^{1} g(s) x(s) d s$, where $t \in[0,1], 0<\alpha<1$, and $f$ is a given function. We point out that such a kind of initial conditions or nonlocal restrictions could play an interesting role in the applications of the mentioned model. The results obtainded are applied to an example.
\end{abstract}

\section{Introduction}

Recently it have been proved that the differential models involving nonlocal derivatives of fractional order arise in many engineering and scientific disciplines as the mathematical modeling of systems and processes in many fields, for instance, physics, chemistry, aerodynamics, electrodynamics of complex medium, polymer rheology, and so forth (see [16]). In fact, such models can be considered as an efficient alternative to the classical nonlinear differential models to simulate many complex processes (see [7]). For instance, fractional differential equations are an excellent tool to describe hereditary properties of viscoelastic materials and, in general, to simulate the dynamics of many processes on anomalous media. Theory of fractional differential equations has been extensively studied by several authors as Delbosco and Rodino [8], Kilbas et al. [6], Lakshmikantham et al. [9-11], and also see $[2,12-16]$. 

conditions

Recently Mophou and N'Guérékata [17], studied the Cauchy problem with nonlocal

$$
\begin{gathered}
D^{q} x(t)=A x(t)+t^{n} f(t, x(t), B x(t)), \quad t \in[0, T], n \in Z^{+}, \\
x(0)+g(x)=x_{0},
\end{gathered}
$$

in general Banach space $X$ with $0<q<1$, and $A$ is the infinitesimal generator of a $C_{0^{-}}$ semigroup of bounded linear operators. By means of the Krasnoselskii's Theorem, existence of solutions was also obtained.

Subsequently several authors have investigated the problem for different types of nonlinear differential equations and integrodifferential equations including functional differential equations in Banach spaces.

Very recently N'Guérékata [2,18] discussed the existence of solutions of fractional abstract differential equations with nonlocal initial condition. The nonlocal Cauchy problem is discussed by authors in [15] using the fixed-point concepts. Tidke [19] studied the nonfractional mixed Volterra-Fredholm integrodifferential equations with nonlocal conditions using Leray-Schauder Theorem.

Motivated by the above mentioned works in this manuscript we discuss the existence and the uniqueness of the solution for the following fractional integrodifferential equation with nonlocal integral initial condition in Banach Space:

$$
\begin{gathered}
\frac{d^{\alpha} x(t)}{d t^{\alpha}}=f\left(t, x(t), \int_{0}^{t} k(t, s, x(s)) d s, \int_{0}^{1} h(t, s, x(s)) d s\right), \\
x(0)=\int_{0}^{1} g(s) x(s) d s
\end{gathered}
$$

where $t \in J=[0,1], 0<\alpha<1, g(t) \in(0,1], g \in L^{1}\left([0,1], \mathbb{R}_{+}\right), x \in Y=C(J, E)$ is a continuous function on $J$ with values in the Banach space $E$ and $\|x\|_{Y}=\max _{t \in J}\|x(t)\|_{E}$, and $f: J \times E \times$ $E \times E \rightarrow E, k: D \times E \rightarrow E$, and $h: D_{0} \times E \rightarrow E$ are continuous $E$-valued functions. Here $D=\left\{(t, s) \in \mathbb{R}^{2}: 0 \leq s \leq t \leq 1\right\}$, and $D_{0}=J \times J$. The operator $d^{\alpha} / d t^{\alpha}$ denotes the Caputo fractional derivative of order $\alpha$.

For the sake of the shortness let

$$
K x(t)=\int_{0}^{t} k(t, s, x(s)) d s, \quad H x(t)=\int_{0}^{1} h(t, s, x(s)) d s .
$$

The paper is organized as follows. In Section 2, some definitions, lemmas and preliminary results are introduced to be used in the sequel. Section 3 will involve the assumptions, main results and proofs of existence problem of (1.2), together with a nonlocal initial condition. Finally an example is presented.

\section{Preliminaries}

Let $E$ be a real Banach space and $\theta$ the zero element of $E$. Let $L^{1}([0,1], E)$ be the Banach space of measurable functions $x:[0,1] \rightarrow E$ which are Lebesgue integrable, equipped with the 
norm $\|x\|_{L^{1}}=\int_{0}^{1}\|x(s)\| d s$. We will use the following notation $R^{+}=(0, \infty)$, and $R_{+}=[0, \infty)$. A function $x \in C([0,1], E)$ is called a solution of (1.2) if it satisfies (1.2).

Definition 2.1. A real function $f(t)$ is said to be in the space $C_{\alpha}, \alpha \in R$ if there exists a real number $p>\alpha$, such that $f(t)=t^{p} g(t)$, where $g \in C[0, \infty)$, while $f(t)$ is said to be in the space $C_{\alpha}^{m}$ if and only if $f^{(m)} \in C_{\alpha}, m \in N$.

Definition 2.2. The fractional (arbitrary) order Riemann-Liouville integral (on the right and on the left) of the function $f \in L^{1}\left([a, b], R_{+}\right)$of order $\alpha, a, b \in R_{+}$is defined by

$$
I_{a}^{\alpha} f(t)=\frac{1}{\Gamma(\alpha)} \int_{a+}^{t} \frac{(t-s)^{\alpha-1}}{\Gamma(\alpha)} f(s) d s, \quad I_{b-}^{\alpha} f(t)=\frac{1}{\Gamma(\alpha)} \int_{t}^{b} \frac{(s-t)^{\alpha-1}}{\Gamma(\alpha)} f(s) d s,
$$

where $\Gamma$ is the Gamma function of Euler.

When $a=0$, we write $I^{\alpha} f(t)=I_{0+}^{\alpha} f(t)=f * \phi_{\alpha}(t)$, where $\phi_{\alpha}(t)=t^{\alpha-1} / \Gamma(\alpha)$ for $t>0$, $\phi_{\alpha}(t)=0$ for $t \leq 0$, and $*$ represents the Convolution of Laplace. Then, it is well known that $\phi_{\alpha}(t) \rightarrow \delta(t)$ as $\alpha \rightarrow 0$, where $\delta$ is the Delta function.

Definition 2.3. The Riemann-Liouville fractional integral operator of order $\alpha>0$, of a function $f \in C_{\mu}, \mu \geq-1$ is defined as

$$
\begin{gathered}
I^{\alpha} f(t)=\phi_{\alpha} * f(t), \quad \alpha>0, t>0, \\
I^{0} f(x)=f(x) .
\end{gathered}
$$

Definition 2.4. The Caputo's derivative of fractional order $\alpha>0$ for a suitable function $f(t)$ is defined by

$$
\left({ }^{c} D^{\alpha} f\right)(t)=\frac{1}{\Gamma(n-\alpha)} \int_{0}^{t} \frac{f^{(n)}(s)}{(t-s)^{\alpha-n+1}} d s, \quad n-1<\alpha<n, n=[\alpha]+1,
$$

where $[\alpha]$ denotes the integer part of real number $\alpha$.

It is obvious that the Caputo's derivative of a constant is equal to 0 .

Lemma 2.5. Let $\alpha>0$ and $n=[\alpha]+1$. Then

$$
I^{\alpha}\left({ }^{c} D^{\alpha} f\right)(t)=f(t)-\sum_{k=0}^{n-1} \frac{f^{(k)}(0)}{k !} t^{k}
$$

Lemma 2.6. If $Q(\tau, \alpha)=\Gamma(\alpha) I_{1-}^{\alpha} g(\tau)=\int_{\tau}^{1} g(s)(s-\tau)^{\alpha-1} d s$ for $\tau \in[0,1]$, and if $g \in L^{1}\left([0,1], R_{+}\right)$ satisfies $0 \leq g(s) \leq 1$ for $0 \leq s \leq 1$ and $\alpha>0$, then

$$
\frac{Q(\tau, \alpha)}{\Gamma(\alpha)}<e, \quad \frac{\int_{0}^{t}(t-s)^{\alpha-1} d s}{\Gamma(\alpha)}<e .
$$


Proof. A direct computation shows

$$
\begin{aligned}
\frac{Q(\tau, \alpha)}{\Gamma(\alpha)} & =\frac{\int_{\tau}^{1} g(s)(s-\tau)^{\alpha-1} d s}{\int_{0}^{\infty} s^{\alpha-1} e^{-s} d s}=\frac{\int_{\tau}^{1}(s-\tau)^{\alpha-1} d s}{\int_{0}^{\infty} s^{\alpha-1} e^{-s} d s} \\
& =\frac{\int_{0}^{1-\tau} s^{\alpha-1} d s}{\int_{0}^{\infty} s^{\alpha-1} e^{-s} d s} \leq \frac{e \int_{0}^{1-\tau} s^{\alpha-1} e^{-s} d s}{\int_{0}^{\infty} s^{\alpha-1} e^{-s} d s}<e, \\
\frac{\int_{0}^{t}(t-s)^{\alpha-1} d s}{\Gamma(\alpha)} & =\frac{\int_{0}^{t} s^{\alpha-1} d s}{\int_{0}^{\infty} s^{\alpha-1} e^{-s} d s} \leq \frac{e \int_{0}^{t} s^{\alpha-1} e^{-s} d s}{\int_{0}^{\infty} s^{\alpha-1} e^{-s} d s}<e .
\end{aligned}
$$

Theorem 2.7 (Krasnoselkii). Let X be a Banach space, let $S$ be a bounded closed convex subset of $X$ and let $A, B$ be maps of $S$ into $X$ such that $A x+B y \in S$ for every pair $x, y \in S$. If $A$ is completely continuous and $B$ is a contraction then the equation $A x+B x=x$ has a solution on $S$.

\section{Main Results}

We assume the following.

(A1) If $f \in C([0,1] \times E \times E \times E, E)$ and a nonnegative, bounded $p_{f} \in L^{1}\left([0,1], R^{+}\right)$, there exist $M>0, p_{f}(t) \leq M$ for $t \in[0,1]$ such that

$$
\|f(t, x, K x, H x)\| \leq p_{f}(t)\|x\| \quad \text { for } x \in E .
$$

(A2) There exist positive constants $L_{1}, L_{2}$, and $L$ such that

$$
\left\|f\left(t, x_{1}, y_{1}, z_{1}\right)-f\left(t, x_{2}, y_{2}, z_{2}\right)\right\| \leq L_{1}\left(\left\|x_{1}-x_{2}\right\|+\left\|y_{1}-y_{2}\right\|+\left\|z_{1}-z_{2}\right\|\right)
$$

for all $x_{1}, y_{1}, z_{1}, x_{2}, y_{2}, z_{2} \in Y, L_{2}=\max _{t \in J}\|f(t, 0,0,0)\|$, and $L=\max \left\{L_{1}, L_{2}\right\}$.

(A3) There exist positive constants $N_{1}, N_{2}$, and $N$ such that

$$
\left\|k\left(t, s, x_{1}\right)-k\left(t, s, x_{2}\right)\right\| \leq N_{1}\left\|x_{1}-x_{2}\right\|
$$

for all $x_{1}, x_{2} \in Y, N_{2}=\max _{(t, s) \in D}\|k(t, s, 0)\|$, and $N=\max \left\{N_{1}, N_{2}\right\}$.

(A4) There exist positive constants $C_{1}, C_{2}$, and $C$ such that

$$
\left\|h\left(t, s, x_{1}\right)-h\left(t, s, x_{2}\right)\right\|<C_{1}\left\|x_{1}-x_{2}\right\|
$$

for all $x_{1}, x_{2} \in Y, C_{2}=\max _{(t, s) \in D_{0}}\|h(t, s, 0)\|$, and $C=\max \left\{C_{1}, C_{2}\right\}$.

(A5) $p=(L / \Gamma(\alpha+1))(1+C+N /(\alpha+1))$ is such that $0 \leq p<1$.

Firstly, we obtain the following lemmas to prove the main results on the existence of solutions to (1.2). 
Lemma 3.1. If (A1) holds with $\mu=\int_{0}^{1} g(s) d s$, then the problem (1.2) is equivalent to the following equation:

$$
x(t)=\frac{1}{(1-\mu) \Gamma(\alpha)} \int_{0}^{1} Q(\tau, \alpha) f(\tau, x(\tau), K x(\tau), H x(\tau)) d \tau+\phi_{\alpha}(t) * f(t, x(t), K x(t), H x(t)) .
$$

Proof. By Lemma 2.5 and (1.2), we have

$$
x(t)=x(0)+\frac{1}{\Gamma(\alpha)} \int_{0}^{t}(t-s)^{\alpha-1} f(s, x(s), K x(s), H x(s)) d s .
$$

Therefore,

$$
\begin{aligned}
x(0) & =\int_{0}^{1} g(s) x(s) d s \\
& =\int_{0}^{1} g(s) d s x(0)+\frac{1}{\Gamma(\alpha)} \int_{0}^{1} g(s) \int_{0}^{s}(s-\tau)^{\alpha-1} f(\tau, x(\tau), K x(\tau), H x(\tau)) d \tau d s .
\end{aligned}
$$

So,

$$
\begin{aligned}
x(0) & =\frac{1}{(1-\mu) \Gamma(\alpha)} \int_{0}^{1} f(\tau, x(\tau), K x(\tau), H x(\tau))\left[\int_{\tau}^{1}(s-\tau)^{\alpha-1} g(s) d s\right] d \tau \\
& =\frac{1}{(1-\mu) \Gamma(\alpha)} \int_{0}^{1} Q(\tau, \alpha) f(\tau, x(\tau), K x(\tau), H x(\tau)) d \tau,
\end{aligned}
$$

and then

$$
x(t)=\frac{1}{(1-\mu) \Gamma(\alpha)} \int_{0}^{1} Q(\tau, \alpha) f(\tau, x(\tau), K x(\tau), H x(\tau)) d \tau+\phi_{\alpha}(t) * f(t, x(t), K x(t), H x(t)) .
$$

Conversely, if $x$ is a solution of (3.5), then for every $t \in[0,1]$, according to Definition 2.4 we have

$$
\begin{aligned}
&{ }^{c} D^{\alpha} x(t)={ }^{c} D^{\alpha}\left[\frac{1}{(1-\mu) \Gamma(\alpha)} \int_{0}^{1} Q(\tau, \alpha) f(\tau, x(\tau), K x(\tau), H x(\tau)) d \tau\right. \\
&\left.+\phi_{\alpha}(t) * f(t, x(t), K x(t), H x(t))\right] \\
&= \theta+{ }^{c} D^{\alpha}\left(I^{\alpha} f(t, x(t), K x(t), H x(t))\right) \\
&= f(t, x(t), K x(t), H x(t)) .
\end{aligned}
$$

It is obvious that $x(0)=\int_{0}^{1} g(s) x(s) d s$. This completes the proof. 
Lemma 3.2. If (A3) and (A4) are satisfied, $K, H$ are defined in (A), then the conditions

$$
\begin{gathered}
\|K x(t)\| \leq t\left(N_{1}\|x\|+N_{2}\right), \\
\left\|K x_{1}(t)-K x_{2}(t)\right\| \leq N_{1} t\left\|x_{1}-x_{2}\right\|, \\
\|H x(t)\| \leq\left(C_{1}\|x\|+C_{2}\right), \\
\left\|H x_{1}(t)-H x_{2}(t)\right\| \leq C_{1}\left\|x_{1}-x_{2}\right\|,
\end{gathered}
$$

are satisfied for any $t \in J$, and $x, x_{1}, x_{2} \in Y$.

Proof. By (A3), we have

$$
\begin{aligned}
\|K x(t)\| & \leq \int_{0}^{t}\|k(t, s, x(s))\| d s \\
& =\int_{0}^{t}\|k(t, s, x(s))-k(t, s, 0)+k(t, s, 0)\| d s \\
& \leq \int_{0}^{t}\|k(t, s, x(s))-k(t, s, 0)\| d s+\int_{0}^{t}\|k(t, s, 0)\| d s \\
& \leq N_{1} t\|x\|+N_{2} t \leq\left(N_{1}\|x\|+N_{2}\right) .
\end{aligned}
$$

On the other hand,

$$
\begin{aligned}
\left\|K x_{1}(t)-K x_{2}(t)\right\| & \leq \int_{0}^{t}\left\|k\left(t, s, x_{1}(s)\right)-k\left(t, s, x_{2}(s)\right)\right\| d s \\
& \leq N_{1} \int_{0}^{t}\left\|x_{1}(s)-x_{2}(s)\right\| d s \\
& \leq N_{1} t\left\|x_{1}-x_{2}\right\| .
\end{aligned}
$$

Similarly, for the other conditions, we use assumption (A4), to get

$$
\begin{gathered}
\|H x(t)\| \leq \int_{0}^{1}\|h(t, s, x(s))\| d s \leq\left(C_{1}\|x\|+C_{2}\right), \\
\left\|K x_{1}(t)-K x_{2}(t)\right\| \leq C_{1}\left\|x_{1}-x_{2}\right\| .
\end{gathered}
$$

Theorem 3.3. If (A1)-(A5) are satisfied, then the fractional integrodifferential equation (1.2) has a unique solution continuous in $\mathrm{J}$.

Proof. We use the Banach contraction principle to prove the existence and uniqueness of the solution to (1.2). Let $B_{r}=\{x \in Y:\|x\| \leq r\} \subseteq Y$, where $r \geq(e M /(1-\mu)+(L / \Gamma(\alpha+1))(1+$ $C+N /(\alpha+1)))$ and define the operator $\Psi$ on the Banach space $Y$ by

$$
\begin{aligned}
\Psi(x(t))= & \frac{1}{(1-\mu) \Gamma(\alpha)} \int_{0}^{1} Q(\tau, \alpha) f(\tau, x(\tau), K x(\tau), H x(\tau)) d \tau \\
& +\frac{1}{\Gamma(\alpha)} \int_{0}^{t}(t-s)^{\alpha-1} f(s, x(s), K x(s), H x(s)) d s
\end{aligned}
$$


Firstly, we show that the operator $\Psi$ maps $B_{r}$ into itself. By using (A1) and triangle inequality, we have

$$
\begin{aligned}
\|\Psi(x(t))\| \leq & \frac{e M}{1-\mu}\|x\|+\frac{1}{\Gamma(\alpha)}\left\|\int_{0}^{t}(t-s)^{\alpha-1} f(s, x(s), K x(s), H x(s)) d s\right\| \\
\leq & \frac{e M}{1-\mu}\|x\|+\frac{1}{\Gamma(\alpha)} \int_{0}^{t}(t-s)^{\alpha-1}\|f(s, x(s), K x(s), H x(s))\| d s \\
\leq & \frac{e M}{1-\mu}\|x\| \\
& +\frac{1}{\Gamma(\alpha)} \int_{0}^{t}(t-s)^{\alpha-1}\|f(s, x(s), K x(s), H x(s))-f(s, 0,0,0)+f(s, 0,0,0)\| d s \\
\leq & \frac{e M}{1-\mu}\|x\|+\frac{1}{\Gamma(\alpha)} \int_{0}^{t}(t-s)^{\alpha-1}\|f(s, x(s), K x(s), H x(s))-f(s, 0,0,0)\| d s \\
& +\frac{1}{\Gamma(\alpha)} \int_{0}^{t}(t-s)^{\alpha-1}\|f(s, 0,0,0)\| d s .
\end{aligned}
$$

Now, if (A2) is satisfied, then

$$
\begin{aligned}
\|\Psi(x(t))\| \leq & \frac{e M}{1-\mu}\|x\|+\frac{L_{1}}{\Gamma(\alpha)} \int_{0}^{t}(t-s)^{\alpha-1}(\|x(s)\|+\|K x(s)\|+\|H x(s)\|) d s \\
& +\frac{L_{2}}{\Gamma(\alpha)} \int_{0}^{t}(t-s)^{\alpha-1} d s \\
\leq & \frac{e M}{1-\mu}\|x\|+\frac{L_{1}}{\Gamma(\alpha)} \int_{0}^{t}(t-s)^{\alpha-1}\|x(s)\| d s+\frac{L_{1}}{\Gamma(\alpha)} \int_{0}^{t}(t-s)^{\alpha-1}\|K x(s)\| d s \\
& +\frac{L_{1}}{\Gamma(\alpha)} \int_{0}^{t}(t-s)^{\alpha-1}\|H x(s)\| d s+\frac{L_{2}}{\Gamma(\alpha)} \int_{0}^{t}(t-s)^{\alpha-1} d s .
\end{aligned}
$$

Using Lemma 3.2 and (A3), we have

$$
\begin{aligned}
\|\Psi(x(t))\| \leq & \frac{e M}{1-\mu}\|x\|+\frac{L_{1}}{\Gamma(\alpha+1)} t^{\alpha}\|x\|+\frac{L_{1}}{\Gamma(\alpha)}\left(N_{1}\|x\|+N_{2}\right) \int_{0}^{t}(t-s)^{\alpha-1}(s) d s \\
& +\frac{L_{1}}{\Gamma(\alpha+1)}\left(C_{1}\|x\|+C_{2}\right) t^{\alpha}+\frac{L_{2}}{\Gamma(\alpha+1)} t^{\alpha} \\
\leq & \frac{e M}{1-\mu}\|x\|+\frac{L_{1}}{\Gamma(\alpha+1)} t^{\alpha}\|x\|+\frac{L_{1}}{\Gamma(\alpha+2)} t^{\alpha+1}\left(N_{1}\|x\|+N_{2}\right) \\
& +\frac{L_{1}}{\Gamma(\alpha+1)}\left(C_{1}\|x\|+C_{2}\right) t^{\alpha}+\frac{L_{2}}{\Gamma(\alpha+1)} t^{\alpha} \\
= & \frac{e M}{1-\mu}\|x\|+\frac{L_{1} N_{2}}{\Gamma(\alpha+2)} t^{\alpha+1}+\frac{L_{1} C_{2}}{\Gamma(\alpha+1)} t^{\alpha}+\frac{L_{2}}{\Gamma(\alpha+1)} t^{\alpha} \\
& +\frac{L_{1}}{\Gamma(\alpha+1)} t^{\alpha}\left(1+\frac{N_{1}}{\alpha+1} t+C_{1}\right)\|x\|
\end{aligned}
$$


if $x \in B_{r}$, we have

$$
\begin{aligned}
\|\Psi(x(t))\| \leq & \frac{e M}{1-\mu}\|x\|+\frac{L}{\Gamma(\alpha+1)}\left(1+\frac{N}{\alpha+1}+C\right) \\
& +\frac{L r}{\Gamma(\alpha+1)}\left(1+\frac{N}{\alpha+1}+C\right) \\
\leq & r .
\end{aligned}
$$

Thus $\Psi B_{r} \subset B_{r}$. Next, we prove that $\Psi$ is a contraction mapping. For this, let $x_{1}, x_{2} \in Y$. Applying (A2), we have

$$
\begin{aligned}
& \left\|\Psi x_{1}(t)-\Psi x_{2}(t)\right\| \\
& =\left\|\frac{Q(\tau, \alpha)}{(1-\mu) \Gamma(\alpha)} \int_{0}^{1}\left[f\left(s, x_{1}(\tau), K x_{1}(\tau), H x_{1}(\tau)\right)-f\left(s, x_{2}(\tau), K x_{2}(\tau), H x_{2}(\tau)\right)\right] d \tau\right\| \\
& +\| \frac{1}{\Gamma(\alpha)} \int_{0}^{t}(t-s)^{\alpha-1} f\left(s, x_{1}(s), K x_{1}(s), H x_{1}(s)\right) d s \\
& \quad \quad-\frac{1}{\Gamma(\alpha)} \int_{0}^{t}(t-s)^{\alpha-1} f\left(s, x_{2}(s), K x_{2}(s), H x_{2}(s)\right) d s \| \\
& \leq \frac{e}{(1-\mu)} \int_{0}^{1}\left[L_{1}\left\|x_{1}(\tau)-x_{2}(\tau)\right\|+\left\|K x_{1}(\tau)-K x_{2}(\tau)\right\|+\left\|H x_{1}(\tau)-H x_{2}(\tau)\right\|\right] d \tau \\
& \quad+\frac{L_{1}}{\Gamma(\alpha)}\left(\int_{0}^{t}(t-s)^{\alpha-1}\left\|x_{1}(s)-x_{2}(s)\right\| d s\right. \\
& \left.\quad+\int_{0}^{t}(t-s)^{\alpha-1}\left\|K x_{1}(s)-K x_{2}(s)\right\| d s+\int_{0}^{t}(t-s)^{\alpha-1}\left\|H x_{1}(s)-H x_{2}(s)\right\| d s\right)
\end{aligned}
$$

then using (A3), (A4) and Lemma 3.2, one gets

$$
\begin{aligned}
&\left\|\Psi x_{1}(t)-\Psi x_{2}(t)\right\| \\
& \leq \frac{L_{1} e}{(1-\mu)}\left\|x_{1}-x_{2}\right\|\left(\int_{0}^{1} d \tau+N_{1} \int_{0}^{1} \tau d \tau+C_{1} \int_{0}^{1} d \tau\right) \\
&+\frac{L_{1}}{\Gamma(\alpha)}\left\|x_{1}-x_{2}\right\|\left(\int_{0}^{t}(t-s)^{\alpha-1} d s+N_{1} \int_{0}^{t}(t-s)^{\alpha-1}(s) d s+C_{1} \int_{0}^{t}(t-s)^{\alpha-1} d s\right) \\
& \leq \frac{L_{1} e}{(1-\mu)}\left(1+\frac{N_{1}}{2}+C_{1}\right)\left\|x_{1}-x_{2}\right\|+\frac{L_{1}}{\Gamma(\alpha)}\left(\frac{t^{\alpha}}{\alpha}+\frac{N_{1} \Gamma(\alpha) t^{\alpha+1}}{\Gamma(\alpha+2)}+\frac{C_{1} t^{\alpha}}{\alpha}\right)\left\|x_{1}-x_{2}\right\| \\
&= {\left[\frac{L_{1} e}{(1-\mu)}\left(1+\frac{N_{1}}{2}+C_{1}\right)+\frac{L_{1}}{\Gamma(\alpha+1)}\left(1+C_{1}+\frac{N_{1}}{\alpha+1}(t)\right) t^{\alpha}\right]\left\|x_{1}-x_{2}\right\| } \\
& \leq {\left[\frac{L e}{(1-\mu)}\left(1+\frac{N}{2}+C_{1}\right)+\frac{L}{\Gamma(\alpha+1)}\left(1+C+\frac{N}{\alpha+1}\right)\right]\left\|x_{1}-x_{2}\right\| } \\
& \leq {\left[\frac{L e}{(1-\mu)}\left(1+\frac{N}{2}+C\right)+p\right]\left\|x_{1}-x_{2}\right\| } \\
& \leq \Omega_{C, N, \alpha, \mu, p}\left\|x_{1}-x_{2}\right\|,
\end{aligned}
$$


where $\Omega_{C, N, \alpha, \mu, p}=[(L e /(1-\mu))(1+(N / 2)+C)+p]<1$ depends on the parameter of the problem. Therefore $\Psi$ has a unique fixed-point $x=\Psi(x) \in B_{r}$, which is a solution of (3.5), and hence is a solution of (1.2).

Theorem 3.4. Assume (A1)-(A4) holds. If eM $<1-\mu$, then (1.2) has at least one solution on I.

Proof. Choose $r \geq e M /(1-\mu)+\left\|p_{f}\right\|_{L^{1}} / \Gamma(\alpha+1)$ and consider $B_{r}:\{x \in C:\|x\| \leq r\}$. Now define on $B_{r}$ the operators $A, B$ by

$$
\begin{gathered}
(A x)(t):=\phi_{\alpha}(t) * f(t, x(t), K x(t), H x(t)), \\
(B x)(t):=\frac{1}{(1-\mu) \Gamma(\alpha)} \int_{0}^{1} Q(\tau, \alpha) f(\tau, x(\tau), K x(\tau), H x(\tau)) d \tau .
\end{gathered}
$$

Let us observe that if $x, y \in B_{r}$ then $A x+B y \in B_{r}$. Indeed it is easy to check the inequality

$$
\|A x+B y\| \leq \frac{e M}{(1-\mu)}+\frac{\left\|p_{f}\right\|_{L^{1}}}{\Gamma(\alpha+1)} \leq r .
$$

We can easily show that that $B$ is a contraction mapping. Let $u, v \in B_{r}$. Then

$$
\begin{aligned}
& \|(B u)(t)-(B v)(t)\| \\
& \quad:=\frac{1}{(1-\mu) \Gamma(\alpha)} \int_{0}^{1} Q(\tau, \alpha)\|f(\tau, u(\tau), K u(\tau), H u(\tau))-f(\tau, v(\tau), K v(\tau), H v(\tau))\| d \tau \\
& \quad \leq \frac{e}{(1-\mu)} \int_{0}^{1}\left[L_{1}\|u(\tau)-v(\tau)\|+\|K u(\tau)-K v(\tau)\|+\|H u(\tau)-H v(\tau)\|\right] d \tau \\
& \quad \leq \frac{L_{1} e}{(1-\mu)}\left(1+\frac{N_{1}}{2}+C_{1}\right)\|u-v\| \\
& \leq \Lambda_{N_{1}, C_{1}, L_{1}}\|u-v\|
\end{aligned}
$$

where $\Lambda_{N_{1}, C_{1}, L_{1}}=\left(L_{1} e /(1-\mu)\right)\left(1+\left(N_{1} / 2\right)+C_{1}\right)<1$ depends only on the parameter of the problem and hence $B$ is contraction. Since $x$ is continuous, then $(A x)(t)$ is continuous in view of (A1). Let us now note that $A$ is uniformly bounded on $B_{r}$. This follows from the inequality

$$
\|(A x)(t)\| \leq \frac{\left\|p_{f}\right\|_{L^{1}}}{\Gamma(\alpha+1)}
$$

Now let us prove that $(A x)(t)$ is equicontinuous. 
Let $t_{1}, t_{2} \in J$ and $x \in B_{r}$. Using the fact that $f$ is bounded on the compact set $J \times B_{r}$ (thus $\left.\sup _{(t, s) \in J \times B_{r}}\|f(t, x(s), K x(s), H x(s))\|:=c_{0}<\infty\right)$, we will get

$$
\begin{aligned}
&\left\|A x\left(t_{1}\right)-A x\left(t_{2}\right)\right\|= \frac{1}{\Gamma(q)} \| \int_{0}^{t_{1}}\left(t_{1}-s\right)^{q-1} \int_{0}^{s} f(s, x(s), K x(s), H x(s)) d s \\
& \quad-\int_{0}^{t_{2}}\left(t_{2}-s\right)^{q-1} \int_{0}^{s} f(s, x(s), K x(s), H x(s)) d s \| \\
&=\frac{1}{\Gamma(q)} \| \int_{t_{2}}^{t_{1}}\left(t_{1}-s\right)^{q-1} \int_{0}^{s} f(s, x(s), K x(s), H x(s)) d s \\
& \leq \frac{1}{\Gamma(q)}\left(\left\|\int_{t_{2}}^{t_{1}}\left(t_{1}-s\right)^{q-1} \int_{0}^{s} f(s, x(s), K x(s), H x(s)) d s\right\|\right) \\
&+\frac{1}{\Gamma(q)}\left(\| \int_{0}^{t_{2}}\left(t_{2}-s\right)^{q-1}-\left(t_{1}-s\right)^{q-1}\right) \int_{0}^{s} f(s, x(s), K x(s), H x(s)) d s \| \\
& \leq \frac{c_{0}}{\Gamma(q+1)}\left|2\left(t_{1}-t_{2}\right)^{q}+t_{2}^{q}-t_{1}^{q}\right| \\
& \leq \frac{2 c_{0}}{\Gamma(q+1)}\left|t_{1}-t_{2}\right|^{q},
\end{aligned}
$$

which does not depend on $x$. So $A\left(B_{r}\right)$ is relatively compact. By the Arzela-Ascoli Theorem, $A$ is compact. We now conclude the result of the theorem based on the Krasnoselkii's theorem above.

\section{Example}

Consider the following fractional integrodifferential equation:

$$
\begin{gathered}
{ }^{c} D^{\alpha} x(t)=\frac{e^{-t} x(t)}{\left(9+e^{t}\right)(1+x(t))}+\frac{1}{10} \int_{0}^{t} e^{-(1 / 2) x(s)} d s+\frac{1}{10} \int_{0}^{t} e^{-(1 / 49) x(s)} d s, \quad t \in J=[0,1], \\
x(0)=\int_{0}^{1} \frac{1}{2} x(s) d s,
\end{gathered}
$$


where $0<\alpha \leq 1$. Take $E=R^{+}$. Set $K x(t)=\int_{0}^{t} e^{-(1 / 2) x(s)} d s, H x(t)=\int_{0}^{t} e^{-(1 / 49) x(s)} d s$, $f(t, x, K x, H x)=e^{-t} x /\left(9+e^{t}\right)(1+x)+K x+H x, g(s)=1 / 2, p_{f}(t)=e^{-t} x(t) /\left(9+e^{t}\right)$. Then it is clear that

$$
\begin{aligned}
& f \in C([0,1] \times E \times E \times E, E), \quad p_{f}(t) \leq \frac{1}{10}=M, \\
& p_{f} \in L\left([0,1], R^{+}\right), \quad\|f(t, x, K x, H x)\| \leq p_{f}(x) .
\end{aligned}
$$

So, (A1) is satisfied. Let $x, y \in E$ and $t \in J$. Then we have

$$
\begin{aligned}
& \|K x-K y\|=\left|\int_{0}^{t} e^{-(1 / 2) x(s)} d s-\int_{0}^{t} e^{-(1 / 2) y(s)} d s\right| \leq \frac{1}{2}|x-y| \\
& \begin{aligned}
& \| H x-H y \|=\left|\int_{0}^{t} e^{-(1 / 49) x(s)} d s-\int_{0}^{t} e^{-(1 / 49) y(s)} d s\right| \leq \frac{1}{49}|x-y| \\
&\|f(t, x, K x, H x)-f(t, y, y, H y)\| \\
& \quad=\left|\frac{e^{-t} x}{\left(9+e^{t}\right)(1+x)}-\frac{e^{-t} y}{\left(9+e^{t}\right)(1+y)}+\frac{1}{10}((K x-K y)+(H x-H y))\right| \\
& \quad \leq \frac{e^{-t}|x-y|}{\left(9+e^{t}\right)(1+x)(1+y)}+\frac{1}{10}(\|K x-K y\|+\|H x-H y\|) \\
& \quad \leq \frac{e^{-t}}{9+e^{t}}|x-y|+\frac{1}{10}(\|K x-K y\|+\|H x-H y\|) \\
& \quad \leq \frac{1}{10}(|x-y|+\|K x-K y\|+\|H x-H y\|) .
\end{aligned}
\end{aligned}
$$

Hence the conditions (A1)-(A4) hold with $M=1 / 10, L_{1}=1 / 10, N_{1}=1 / 2, C_{1}=1 / 49$. Choose $r=1$ and $\mu=1 / 2$. Indeed

$$
\begin{gathered}
\frac{L_{1} e}{(1-\mu)}\left(1+\frac{N_{1}}{2}+C_{1}\right)=\frac{149 e}{490}<1, \\
\frac{e M}{1-\mu}=\frac{e}{5}<1 .
\end{gathered}
$$

Further (A5) is satisfied by a suitable choice of $\alpha$. Then by Lemma 3.2 the problem (1.2) has a unique solution on $[0,1]$.

\section{Acknowledgment}

This paper has been partially supported by MICINN (project MTM2010-16499) to which the authors are very thankful. 


\section{References}

[1] M. Caputo, "Linear models of dissipation whose q is almost frequently independent, part II," Journal of the Royal Astronomical Society, vol. 13, pp. 529-539, 1967.

[2] G. M. N'Guérékata, "A Cauchy problem for some fractional abstract differential equation with non local conditions," Nonlinear Analysis: Theory, Methods \& Applications, vol. 70, no. 5, pp. 1873-1876, 2009.

[3] J.-H. He, "Some applications of nonlinear fractional differential equations and their approximation," Bulletin of Science, Technology \& Society, vol. 15, no. 2, pp. 86-90, 1999.

[4] J.-H. He, "Approximate analytical solution for seepage flow with fractional derivatives in porous media," Computer Methods in Applied Mechanics and Engineering, vol. 167, no. 1-2, pp. 57-68, 1998.

[5] R. Hilfer, Ed., Applications of Fractional Calculus in Physics, World Scientific, River Edge, NJ, USA, 2000.

[6] A. A. Kilbas, H. M. Srivastava, and J. J. Trujillo, Theory and Applications of Fractional Differential Equations, vol. 204, North-Holland Mathematics Studies, 2006.

[7] B. Bonilla, M. Rivero, L. Rodríguez-Germá, and J. J. Trujillo, "Fractional differential equations as alternative models to nonlinear differential equations," Applied Mathematics and Computation, vol. 187, no. 1, pp. 79-88, 2007.

[8] D. Delbosco and L. Rodino, "Existence and uniqueness for a nonlinear fractional differential equation," Journal of Mathematical Analysis and Applications, vol. 204, no. 2, pp. 609-625, 1996.

[9] V. Lakshmikantham, "Theory of fractional functional differential equations," Nonlinear Analysis: Theory, Methods \& Applications, vol. 69, no. 10, pp. 3337-3343, 2008.

[10] V. Lakshmikantham and J. V. Devi, "Theory of fractional differential equations in a Banach space," European Journal of Pure and Applied Mathematics, vol. 1, no. 1, pp. 38-45, 2008.

[11] V. Lakshmikantham and A. S. Vatsala, "Basic theory of fractional differential equations," Nonlinear Analysis: Theory, Methods \& Applications, vol. 69, no. 8, pp. 2677-2682, 2008.

[12] B. Ahmad and J. J. Nieto, "Existence results for nonlinear boundary value problems of fractional integrodifferential equations with integral boundary conditions," Boundary Value Problems, vol. 2009, Article ID 708576, 11 pages, 2009.

[13] R. P. Agarwal, V. Lakshmikantham, and J. J. Nieto, "On the concept of solution for fractional differential equations with uncertainty," Nonlinear Analysis: Theory, Methods \& Applications, vol. 72, no. 6, pp. 2859-2862, 2010.

[14] A. Anguraj, P. Karthikeyan, and G. M. N'Guérékata, "Nonlocal Cauchy problem for some fractional abstract integrodifferential equations in Banach space," Communications in Mathematical Analysis, vol. 55 , no. 6, pp. 1-6, 2009.

[15] K. Balachandran and J. Y. Park, "Nonlocal Cauchy problem for abstract fractional semilinear evolution equations," Nonlinear Analysis: Theory, Methods \& Applications, vol. 71, no. 10, pp. 4471-4475, 2009.

[16] L. Byszewski, "Theorems about the existence and uniqueness of solutions of a semilinear evolution nonlocal Cauchy problem," Journal of Mathematical Analysis and Applications, vol. 162, no. 2, pp. $494-$ $505,1991$.

[17] G. M. Mophou and G. M. N'Guérékata, "Existence of the mild solution for some fractional differential equations with nonlocal conditions," Semigroup Forum, vol. 79, no. 2, pp. 315-322, 2009.

[18] G. M. N'Guérékata, "Existence and uniqueness of an integral solution to some Cauchy problem with nonlocal conditions," in Differential \& Difference Equations and Applications, pp. 843-849, Hindawi, New York, NY, USA, 2006.

[19] H. L. Tidke, "Existence of global solutions to nonlinear mixed Volterra-Fredholm integrodifferential equations with nonlocal conditions," Electronic Journal of Differential Equations, vol. 2009, no. 55, 7 pages, 2009. 